\title{
A PROTEASE INHIBITOR, NCO-700, IMPROVES THE CONTRACTILE FUNCTION IN STUNNED CANINE MYOCARDIUM
}

\author{
Hisao Ikeda, M.D., Tameo Oda, M.D., Kazunori Kuwano, M.D. \\ Hiroshi Nakayama, M.D., Takafumi Ueno, M.D., Yoshinori Koga, M.D. \\ and Hironori Toshima, M.D.
}

\begin{abstract}
To explore the role of calcium-dependent protease in the stunned myocardium, open-chest dogs underwent $15 \mathrm{~min}$ of left anterior descending coronary artery occlusion followed by $2 \mathrm{~h}$ of reperfusion. Dogs received a single bolus intravenous injection of either the protease inhibitor NCO-700 $(n=6)$ or saline $(\mathrm{n}=6) 1 \mathrm{~min}$ before reperfusion followed by a 30 -min infusion at the same dose. Regional myocardial function was assessed in terms of systolic wall thickening with an epicardial Doppler probe. The two groups exhibited comparable systolic thickening under baseline conditions and similar degrees of dyskinesis during occlusion. After reperfusion, recovery of contractile function, expressed as a percentage of the baseline value, was significantly greater in NCO-700-treated dogs as than in control dogs: $-14.3 \pm 10.6$ vs $-48.9 \pm 7.2(\mathrm{p}<0.05)$ at $15 \mathrm{~min}$, $10.8 \pm 10.3$ vs $-31.1 \pm 9.0(\mathrm{p}<0.05)$ at $30 \mathrm{~min}, 42.5 \pm 10.1$ vs $-16.4 \pm 9.1(\mathrm{p}<$ $0.005)$ at $1 \mathrm{~h}$, and $47.5 \pm 8.3 \mathrm{vs}-14.9 \pm 9.4(\mathrm{p}<0.001)$ at $2 \mathrm{~h}$. The data suggest that the protease inhibitor markedly improved contractile function in stunned myocardium by inhibiting intracellular protease activity.
\end{abstract}

(Jpn Circ J 1994; 58: 713-719)

$\mathbf{I}^{\mathrm{T}}$ is well established that reperfusion after a brief episode of myocardial ischemia lasting less than $20 \mathrm{~min}$ does not lead to myocardial necrosis, but is associated with a delayed recovery of contractile function. This condition, which is referred to as myocardial stunning, is a mechanical dysfunction that persists after reperfusion despite the absence of irreversible damage, and is distinct from myocardial infarction? Various mechanisms have been proposed for myocardial stunning, including generation of oxygen-derived free radicals, calcium overload,

Key words:

Ischemia

Reperfusion

Oxygen free radicals

Calcium-dependent protease

Stunned myocardium and excitation-contraction uncoupling due to sarcoplasmic reticulum dysfunction.

Bis [ethyl (2R, 3R) -3 -[(S)-methyl -1 -[4 (2, 3, 4 -trimethoxyphenylmethyl) piperazin1-ylcarbonyl] butyl-carbonyl] oxiran-2-carboxylate] sulfate (NCO-700) is a novel inhibitor of protease such as calcium-activated neutral protease (CANP) ${ }^{4}$ and cathepsin? Protease induces degradation of myofibrils when the concentration of calcium in the myocardium is elevated during ischemia and reperfusion6 Furthermore, it has been suggested that calcium-dependent protease mediates major sources of free radicals by conversion of xanthine dehydrogenase to xanthine oxidase? In vivo studies have shown that NCO-700 reduces the infarct size due to coronary artery ligation in rabbit $^{4}$ and in-

(Received March 4, 1993; accepted February 12, 1994)

The Third Department of Internal Medicine, Kurume University School of Medicine, Kurume, Japan

This study was supported in part by a grant from the Kimura Memorial Heart Foundation, Kurume, Japan

Mailing address: Hisao Ikeda, M.D., The Third Department of Internal Medicine, Kurume University School of Medicine, 67 Asahi-machi, Kurume 830, Japan 
hibits the degradation of myofibrillar proteins induced by myocardial ischemia in $\operatorname{dog} 8,9$ However, the role of protease in myocardial stunning is still unclear 10 Therefore, the purposes of this study were to determine whether or not postischemic myocardial dysfunction can be attenuated by pretreatment with NCO-700, and to explore the role of protease in the canine model of stunned myocardium.

\section{MATERIALS AND METHODS}

\section{Experimental Preparation}

All experimental studies were performed according to the Guidelines for Animal Experimentation, based on the Rules and Regulations of the Animal Research Committee of Kurume University School of Medicine. Mongrel dogs of either sex, weighing 12-25 $\mathrm{kg}$, were anesthetized with sodium pentobarbital $(30 \mathrm{mg} / \mathrm{kg})$. The dogs were then ventilated with a tidal volume of $10-20 \mathrm{ml} / \mathrm{kg}$ at a respiratory rate of $15-18$ breaths $/ \mathrm{min}$ using an oxygen-flushed anesthesia machine (Model K-1, Igarashi Ika Kogyo) and a ventilator (Harvard Apparatus). Arterial blood gases were maintained within normal physiologic limits and the body temperature was kept constant using a heating pad. A thoracotomy was performed in the fifth left intercostal space of each dog under sterile conditions and the heart was suspended in a pericardial cradle. Polyethylene catheters were placed in the right atrium through the right atrial appendage for drug infusion. A segment of the left anterior descending (LAD) coronary artery was gently dissected free from the surrounding tissue and a pulsed Doppler flow velocity probe was placed around the LAD artery distal to a balloon occluder. A micromanometer was placed in the femoral artery to monitor aortic pressure. A Doppler ultrasonic wallthickening probe $(10-\mathrm{MHz})$ was positioned in the center of the region to be rendered ischemic, and a second probe was placed in the region of the left circumflex (LCx) coronary artery. An integrated measuring system based on pulsed Doppler (Model VF1, Crystal Biotech, Inc.) simultaneously recorded myocardial thickening, electrocardiogram, and LAD blood flow velocity and continuously recorded these variables on an eight-channel recorder (RECTI-HORIZ-8K, San-ei). Aortic pressure was measured simultaneously.

\section{Experimental Protocol}

NCO-700 (a generous gift from Nippon Chemiphar Research Institute) was dissolved in normal saline solution. The 6 treated dogs received a single bolus infusion of NCO-700 $(20 \mathrm{mg} / \mathrm{kg})$ starting $1 \mathrm{~min}$ before reperfusion. Thereafter, they received a continuous infusion of NCO-700 for 30 min after reperfusion $(20 \mathrm{mg} / \mathrm{kg})$. The 6 control dogs received an equivalent volume of the vehicle (normal saline) under the same protocol.

The LAD coronary artery was occluded for $15 \mathrm{~min}$ and then reperfused. Restoration of blood flow was confirmed by Doppler flow probe measurements. Hemodynamic and wall thickening data were obtained 5 min before coronary occlusion, $10 \mathrm{~min}$ after occlusion, and at 15, 30, 60, and $120 \mathrm{~min}$ after the reperfusion. At the end of the study, the dogs were given a lethal dose of potassium chloride. The heart was removed and cut into $1.0 \mathrm{~cm}$-thick slices in a plane parallel to the atrioventricular groove for 2,3,5triphenyltetrazolium chloride staining. The absence of any myocardial infarction was confirmed in all dogs. Animals which developed lethal arrhythmia during coronary occlusion or following reperfusion were excluded from the present study.

\section{Measurement of Myocardial Function}

Regional myocardial function was assessed using a pulsed Doppler epicardial probe according to the method of Zhu et al!1 Briefly, the pulsed Doppler technique uses a single epicardial transducer to determine systolic myocardial wall displacement by digitally integrating the velocity of myocardial layers which pass through a range-gated sample volume. The beginning and end of systole were determined at the $\mathrm{Q}$ wave on the electrocardiogram, and at the dicrotic notch of the aortic pressure tracing, respectively. The net systolic thickening was defined as the maximal systolic increase in the wall thickness from the end-diastolic value. When paradoxical wall thinning (dyskinesis) persisted for $50 \%$ or more of systole, the maximal extent of wall thinning was subtracted from wall thickening to give a net 


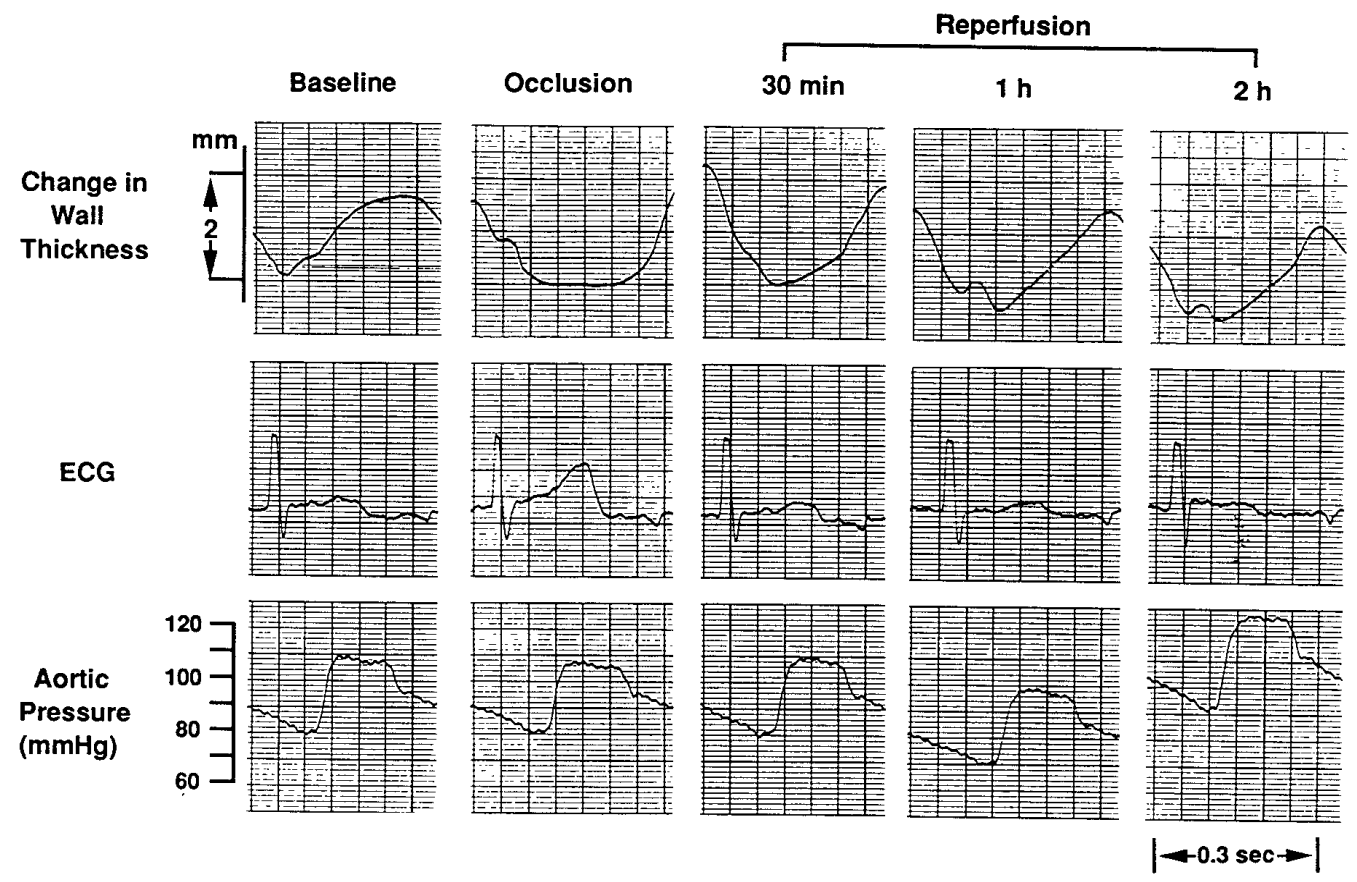

Fig.1. Representative recordings of transmural wall thickening measured sequentially by pulsed Doppler techniques under baseline conditions, during coronary occlusion, $30 \mathrm{~min}, 1 \mathrm{~h}$, and $2 \mathrm{~h}$ after reperfusion. Systolic wall thickening is measured from the Doppler signal as the distance in millimeters between end-systole and enddiastole. Under baseline conditions, thickening fraction was calculated with a simplified formula was as $[1.4 \mathrm{~mm}$ (systolic thickening)/ $7 \mathrm{~mm}$ (range gate depth) $] \times 100=20 \%$. ECG: electrocardiogram.

systolic thickening. Transmural myocardial function was assessed as a percent systolic thickening fraction $(\mathrm{ThF})=$ (transmural net systolic thickening/end-diastolic wall thickness) $\times 100(\%)$. If no paradoxical systolic thinning was not observed until 10 min after coronary occlusion, the dog was excluded from this study because of failure to develop ischemia. Representative recordings of transmural wall thickening at various time points are illustrated in Fig. 1.

\section{Statistical Analysis}

Values are expressed as mean $\pm \mathrm{SE}$. The control and treated dogs were compared using the two-tailed Student's $t$ test for unpaired data. Differences were considered to be significant when the probability was less than 0.05 .

\section{RESULTS}

\section{Excluding Dogs From The Study}

Of the 23 dogs initially anesthetized for the acute experimental protocol, 11 dogs were excluded for the following reasons. Five dogs showed no paradoxical systolic thinning during ischemia. One dog died of ventricular tachycardia during ischemia. Four of the control dogs and 1 of the treated dogs died of ventricular fibrillation following reperfusion. Thus, analysis of data was carried out in 6 control and 6 treated dogs. There was no significant difference between the control and the treated dogs with respect to the incidence of arrhythmias during coronary artery occlusion or subsequent reperfusion. However, the low incidence of ventricular fibrillation and the relatively small sample sizes in our study preclude definitive conclusions.

\section{Hemodynamics and Wall Thickening in Con-} trol and NCO-700-Treated Dogs

The hemodynamic data in the control and NCO-700-treated dogs are presented in Table I. At baseline, occlusion, and reperfusion, there were no significant differences between the control and treated groups with respect to heart rate, systolic or diastolic arterial pressure, and rate-pressure product. Systolic ThF in the LAD artery region is presented as an absolute value in Table I and as a percentage of the baseline 
TABLE I HEMODYNAMIC AND WALL THICKENING DATA IN CONTROL AND NCO-700 TREATED DOGS

\begin{tabular}{|c|c|c|c|c|c|c|}
\hline & \multirow{2}{*}{ Baseline } & \multirow{2}{*}{ Occlusion } & \multicolumn{4}{|c|}{ Reperfusion } \\
\hline & & & $15 \mathrm{~min}$ & $30 \mathrm{~min}$ & $1 \mathrm{~h}$ & $2 h$ \\
\hline \multicolumn{7}{|c|}{ Heart Rate (beats/min) } \\
\hline Control & $146 \pm 3$ & $149 \pm 3$ & $146 \pm 5$ & $146 \pm 6$ & $147 \pm 5$ & $149 \pm 5$ \\
\hline$N C O-700$ & $148 \pm 5$ & $148 \pm 3$ & $149 \pm 6$ & $151 \pm 6$ & $149 \pm 6$ & $147 \pm 4$ \\
\hline \multicolumn{7}{|c|}{ Systolic Arterial Pressure $(\mathrm{mmHg})$} \\
\hline Control & $127 \pm 4$ & $126 \pm 4$ & $127 \pm 4$ & $127 \pm 4$ & $128 \pm 4$ & $127 \pm 5$ \\
\hline NCO-700 & $126 \pm 4$ & $128 \pm 4$ & $127 \pm 4$ & $126 \pm 3$ & $125 \pm 4$ & $128 \pm 5$ \\
\hline \multicolumn{7}{|c|}{ Diastolic Arterial Pressure $(\mathrm{mmHg})$} \\
\hline Control & $83 \pm 5$ & $82 \pm 4$ & $82 \pm 5$ & $82 \pm 5$ & $82 \pm 4$ & $83 \pm 5$ \\
\hline$N C O-700$ & $83 \pm 3$ & $81 \pm 4$ & $82 \pm 4$ & $82 \pm 4$ & $82 \pm 3$ & $84 \pm 4$ \\
\hline \multicolumn{7}{|l|}{$R P P$} \\
\hline Control & $18.6 \pm 0.5$ & $18.8 \pm 0.5$ & $18.9 \pm 1.1$ & $18.7 \pm 0.6$ & $18.7 \pm 0.7$ & $18.8 \pm 0.7$ \\
\hline NCO-700 & $18.7 \pm 0.9$ & $19.0 \pm 0.9$ & $18.8 \pm 0.6$ & $19.1 \pm 1.1$ & $18.7 \pm 1.0$ & $18.8 \pm 0.9$ \\
\hline \multicolumn{7}{|l|}{$\% T h F(L A D)$} \\
\hline Control & $23.3 \pm 1.1$ & $-15.8 \pm 1.5$ & $-11.2 \pm 1.5$ & $-7.0 \pm 1.8$ & $-3.8 \pm 2.2$ & $-3.4 \pm 2.2$ \\
\hline NCO-700 & $22.1 \pm 1.8$ & $-16.2 \pm 1.6$ & $-2.4 \pm 2.1^{a}$ & $3.3 \pm 2.3^{a}$ & $8.9 \pm 1.9^{b}$ & $10.1 \pm 1.6^{c}$ \\
\hline \multicolumn{7}{|l|}{$\% T h F(L C x)$} \\
\hline Control & $17.9 \pm 1.1$ & $18.7 \pm 1.0$ & $18.2 \pm 1.2$ & $18.2 \pm 1.2$ & $16.8 \pm 1.1$ & $16.8 \pm 0.8$ \\
\hline NCO-700 & $17.7 \pm 1.2$ & $18.7 \pm 1.3$ & $17.4 \pm 1.5$ & $17.1 \pm 1.2$ & $16.2 \pm 1.1$ & $16.2 \pm 1.1$ \\
\hline
\end{tabular}

Data are means $\pm S E . R P P:$ rate-pressure product (heart rate $\times$ systolic blood pressurel1,000), \% ThF (LAD): percent thickening fraction in left anterior descending coronary artery territory, \% ThF (LCX): percent thickening fraction in left circumflex coronary artery territory. ${ }^{a} p<0.01,{ }^{b} p<0.005,{ }^{c} p<0.001$ vs control.

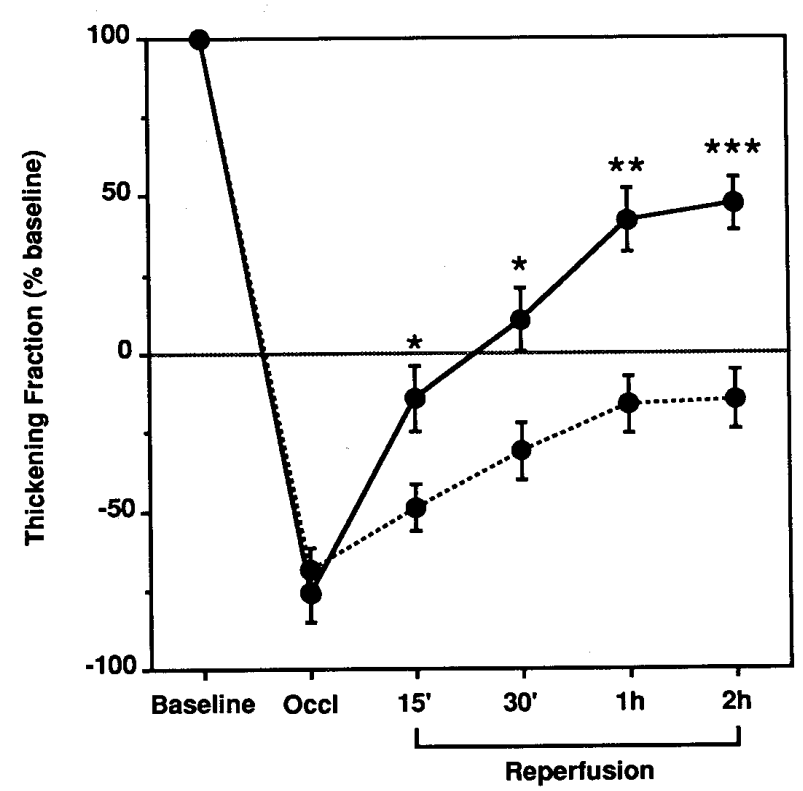

Fig.2. Systolic thickening fraction in the ischemic-reperfused region after coronary occlusion (Occl) and at selected times after reperfusion in control (dashed line) and treated (continuous line) groups. Thickening fraction is expressed as a percent of percentage of the baseline value measured before coronary occlusion. $* \mathrm{p}<0.05$, ${ }^{* *} \mathrm{p}<0.005,{ }^{* * *} \mathrm{p}<0.001$ vs control. value in Fig. 2. Baseline systolic ThF averaged $23.3 \pm 1.1 \%$ in the control and $22.1 \pm$ $1.8 \%$ in the treated dogs (NS). The extent of paradoxical systolic thinning during occlusion was comparable in the control and NCO-700-treated dogs $(-15.8 \pm 1.5$ vs $-16.2 \pm 1.6 \%$, NS). In the control dogs, although there was a gradual improvement in the contractile function following reperfusion, as compared with baseline values, the previously ischemic region remained still dyskinetic $2 \mathrm{~h}$ after reperfusion. In the NCO-700-treated dogs, the recovery of function after reperfusion was substantially greater (Fig. 2). ThF, expressed as a percentage of the baseline values, was $-14.3 \pm 10.6$ in the treated dogs vs $-48.9 \pm 7.2$ in the control $(\mathrm{p}<0.05)$ at $15 \mathrm{~min}$ after reperfusion, $10.8 \pm 10.3$ vs $-31.1 \pm 9.0(\mathrm{p}<0.05)$ at 30 $\min , 42.5 \pm 10.1$ vs $-16.4 \pm 9.1(\mathrm{p}<0.005)$ at $1 \mathrm{~h}$, and $47.5 \pm 8.3$ vs $-14.9 \pm 9.4(\mathrm{p}<0.001)$ at $2 \mathrm{~h}$ after reperfusion. Similar differences were observed when the statistical analysis was performed using the absolute values of ThF (Table I). In the $\mathrm{LCx}$ region, there were no significant differences in $\mathrm{ThF}$ be- 
tween the two groups throughout the entire experiment (Table I).

\section{DISCUSSION}

The present study demonstrates that a protease inhibitor, NCO-700, markedly attenuates postischemic contractile dysfunction. After $2 \mathrm{~h}$ of reperfusion, previously ischemic myocardium had recovered to approximately one-half of its baseline systolic function in dogs that had been treated with NCO-700, but remained dyskinetic in untreated control dogs. These observations suggest that intracellular protease plays an important role in the pathogenesis of regional postischemic myocardial dysfunction caused by a $15 \mathrm{~min}$ coronary occlusion followed by reperfusion. This finding supports the results of a recent study in which leupeptin, a cysteine-protease inhibitor, had a protective effect against myocardial stunning in Langendorff-perfused hearts ${ }^{10}$

Although the pathogenesis of myocardial stunning has not been fully determined, oxyradical generation, calcium overload, and excitation-contraction uncoupling have each been proposed as most likely contributing factors? 3 Oxygen species can cause enzyme inactivation and lipid peroxidation during ischemia and reperfusion. This could induce damage to the sarcolemma and the sarcoplasmic reticulum, resulting in an increased transsarcolemmal calcium influx and a cellular calcium overload, especially under conditions of re-flow. An impaired calcium homeostasis could in turn lead to excitationcontraction uncoupling. Indeed, several studies have demonstrated that oxygen free radical scavengers, such as superoxide dismutase and catalase, significantly enhance the recovery of the stunned myocardium, and suggest that cytotoxic oxygen metabolites, including the superoxide anion, hydrogen peroxide and hydroxyl radical, play an important role in the genesis of myocardial stunning 12-14 Moreover, Bolli et al., using electron paramagnetic resonance spectroscopy, demonstrated that the myocardial production of free radicals begins during coronary occlusion and further increases after reperfusion in canine stunned myocardium 15 In a subsequent study, 16 they found that superoxide dismutase plus catalase suppressed the production of free radicals, indicating that these radicals were derived from the univalent reduction of oxygen.

The xanthine oxidase inhibitor allopurinol has also been shown to inhibit uric acid production during ischemia and reperfusion, and to produce a marked improvement in the functional recovery of stunned myocardium. These data suggest that xanthine oxidase is a source of free radicals that contribute to postischemic dysfunction in the dog.7 In this regard, McCord proposed that elevated cytosolic calcium concentration activates a protease which is capable of converting xanthine dehydrogenase to xanthine oxidase during ischemia? Concomitantly, an elevated concentration of AMP, due to the depletion of ATP during ischemia, is catabolized to adenosine, inosine, and hypoxanthine. The increased hypoxanthine serves as a purine substrate for the xanthine dehydrogenase or xanthine oxidase, leading to further free radical generation in reoxygenated tissues? Therefore, the results of the present study suggest that the beneficial effect of the protease inhibitor NCO-700 on myocardial stunning may be associated with the inhibition of the conversion of xanthine dehydrogenase to xanthine oxidase in microvascular endothelial cells, which thereby reduces oxygen free radical generation, since this agent has been shown to scavenge free radicals generated from the xanthine-xanthine oxidase system 18 However, since we did not attempt to examine the direct effect of NCO700 on either the xanthine dehydrogenasexanthine oxidase system or on free radical formation in the present study, further studies are required.

It is also possible that NCO-700 directly protects against the increase in intracellular protease activity, such as of $\mathrm{CANP}^{4}$ and cathepsin, in endothelial and myocardial cells, if intracellular concentrations of calcium increase during ischemia and reperfusion. A previous study used endothelial cell monolayers to demonstrate that intracellular calcium significantly increased during hypoxia and decreased to reach the normal value of resting cells during the reoxygenation 19 Furthermore, in a recent study using nuclear magnetic resonance spectroscopy which directly measured serial changes in the intracellular concentration of calcium in per- 
fused heart that had been subjected to 20 min of ischemia followed by reperfusion, the intracellular concentration of calcium significantly increased about three fold at 15-20 min and was significantly elevated during the first 5 min after reperfusion, which reflected an increase in the concentration of intracellular calcium of $1 \mu \mathbf{M}^{20}$ On the other hand, CANP has been shown to require more than $\mu \mathrm{M}$ concentrations of calcium for maximum activity ${ }^{21}$ These data suggest the possibility that transient calcium overload may increase intracellular CANP activity, leading to myocardial contractile dysfunction. Therefore, the present study and others ${ }^{10}$ suggest that the preventive effect of protease inhibitor on myocardial stunning may be closely associated with the inhibition of CANP in tissue cells.

Cathepsins have been shown to be released from lysosomes into cytoplasm during ischemia 22 degrading myofibrillar proteins of cardiac muscles. Since NCO-700 is a potent inhibitor of cathepsin, cathepsin may also play a role on myocardial stunning. However, in a very recent study Matsumura et al proposed that lysosomal protease does not play an important role in stunning 10 Thus, although CANP is more likely to contribute to the stunning process, the precise mechanism of the role of CANP in myocardial stunning awaits further investigation.

Collateral flows during ischemia may result in large differences in recovery of contractile function. Previous studies by Bolli et al. using microspheres technique indicated that the presence of hypokinesis during ischemia was associated with rapid recovery after reperfusion, but there was no relation between ischemic and postischemic dysfunction when dyskinesis was present during occlusion, implying that functional recovery in stunned myocardium was closely coupled to collateral flow ${ }^{23}$ In this study, wall thickening measurements were obtained $10 \mathrm{~min}$ after coronary occlusion according to the method of Bolli et al. Wall thinning was not defined as paradoxical (dyskinetic) unless it persisted throughout at least $50 \%$ of the duration of systole. Transmural ThF measured $10 \mathrm{~min}$ after coronary occlusion in control dogs averaged $-15.8 \pm 1.5 \%$ (Table I). This value is consistent with the results of previous studies ${ }^{17}$ excluding dogs which showed a lack of dyskinesis during the 15 min coronary occlusion. Accordingly, the influence of collateral flow during ischemia on regional myocardial function may be relatively lower in this study.

Transient myocardial ischemia is frequently observed in patients with unstable angina or acute myocardial infarction with early recanalization which occurs spontaneously or is produced by thrombolytic therapy and coronary angioplasty 24,25 These clinical situations may induce myocardial stunning and left ventricular dysfunction?,26 The present observations suggest that NCO-700 may be able to prevent postischemic myocardial dysfunction under such clinical conditions. When compared with the free radical scavengers like such as superoxide dismutase and catalase, NCO-700 offers the advantages of entering the intracellular space. Therefore, NCO-700 may be suitable for greater clinical application.

\section{Acknowledgment}

We thank the Nippon Chemiphar Research Institute for their generous gift of NCO-700.

\section{REFERENCES}

1. HEYNDRICKX GR, MILLARD RW, McRITCHIE RJ, MAROKO PR, VATNER SF: Regional myocardial functional and electrophysiological alterations after brief coronary artery occlusion in conscious dogs. J Clin Invest 1975; 56: 978-985

2. BRAUNWALD E, KLONER RA: The stunned myocardium: Prolonged, postischemic ventricular dysfunction. Circulation 1982; 66: 1146-1149

3. BOLLI RB: Mechanism of myocardial "stunning". Circulation 1990; 82: 723-738

4. TOYO-OKA T, KAMISHIRO T, MASAKI M, MASAKI T: Reduction of experimentally produced acute myocardial infarction size by a new synthetic inhibitor, NCO-700, against calcium-activated neural protease. Jpn Heart $J$ 1982; 23: $829-834$

5. HIRAO T, HARA K, TAKAHASHI K: Purification and characterization of cathepsin B from monkey skeletal muscle. J Biochem 1984; 95: $871-879$

6. DAYTON WR, SCHOLLMEYER JV: Isolation from porcine cardiac muscle of a $\mathrm{Ca}^{2+}$-activated protease that partially degrades myofibrils. $\mathrm{J} \mathrm{Mol}$ Cell Cardiol 1980; 12: 533-551

7. McCORD JM: Oxygen-derived free radicals in postischemic tissue injury. $N$ Engl $J$ Med 1985; 312: $159-163$

8. HAGA N, ISHIBASHI T, HARA A, ABIKO Y: Effect of NCO-700, an inhibitor of protease, on 
myocardial $\mathrm{pH}$ decreased by coronary occlusion in dogs. Pharmacology 1985; 31: 208-217

9. SASHIDA H, ABIKO Y: Inhibition with NCO700 , a protease inhibitor, of degradation of cardiac myofibrillar proteins during ischemia in dogs. Biochem Pharmacol 1985; 34: 3875-3880

10. MATSUMURA $Y$, KUSUOKA $H$, INOUE $M$, HORI M, KAMADA T: Protective effect of the protease inhibitor leupeptin against myocardial stunning. J Cardiovasc Pharmacol 1993; 22: $135-142$

11. ZHU WX, MYERS ML, HARTLEY CJ, ROBERTS R, BOLLI R: Validation of a single crystal for measurement of transmural and epicardial thickening. $A m$ J Physiol 1986; 251: H1045-H1055

12. GROSS GJ, FARBER NE, HARDMAN HF, WARLTIER DC: Beneficial actions of superoxide dismutase and catalase in stunned myocardium of dogs. Am J Physiol 1986; 250: H372-H377

13. PRZYKLENK K, KLONER RA: Superoxide dismutase plus catalase improve contractile function in the canine model of the "stunned" myocardium. Circ Res 1986; 58: $148-156$

14. JEROUDI MO, TRIANA FJ, PATEL BS, BOLLI R: Effect of superoxide dismutase and catalase, given separately, on myocardial "stunning". $A m J$ Physiol 1990; 259: H889-H901

15. BOLLI R, PATEL BS, JEROUDI MO, LAI EK, McCAY PB: Demonstration of free radical generation in "stunned" myocardium of intact dogs with the use of the spin trap a-phenyl N-tert-butyl nitrone. J Clin Invest 1988; 82: 476-485

16. BOLLI R, JEROUDI MO, PATEL BS, DuBOSE CM, LAI EK, ROBERTS R, McCAY PB: Direct evidence that oxygen-derived free radicals contribute to postischemic myocardial dysfunction in the intact dog. Proc Natl Acad Sci USA 1989; 86: 4695-4699

17. CHARLAT ML, O'NEILL PG, EGAN JM, ABERNETHY DR, MICHAEL LH, MYERS ML, ROBERTS R, BOLLI R: Evidence for a pathogenetic role of xanthine oxidase in the "stunned" myocardium. Am J Physiol 1987; 252: H566-H577

18. TAWARA K, FUJISAWA S, NAKAI K: Effect of NCO-700, an inhibitor of thiol protease, on reactive oxygen production by chemotactic peptidestimulated rabbit peripheral granulocytes. $E x$ perientia 1988; 44: 346-347

19. ARNPULD T, MICHIELS C, ALEXANDRE I, REMACLE J: Effect of hypoxia upon intracellular calcium concentration of human endothelial cells. J Cell Physiol 1992; 152: 215-221

20. MARBAN E, KITAKAZE M, KORETSUNE Y, YUE DT, CHACKO VP, PIKE MM: Quantification of $\left[\mathrm{Ca}^{2+}\right]_{i}$ in perfused hearts. critical evaluation of the 5F-BAPTA and nuclear magnetic resonance method as applied to the study of ischemia and reperfusion. Circ Res 1990; 66: 1255-1267

21. MELLGREN RL: Canine cardiac calcium-dependent proteases; resolution of two forms with different requirements for calcium. FEBS Lett 1980; 109: $129-133$

22. DECKER RS, POOLE R, WIILDENTHAL: Distribution of lysosomal cathepsin $\mathrm{D}$ in normal, ischemic, and starved rabbit cardiac myocytes. Circ Res 1980; 46: 485-494

23. BOLLI R, ZHU WX, THORNBY JI, O'NEILL PG, ROBERTS R: Time course and determinants of recovery of function after reversible ischemia in conscious dogs. Am J Physiol 1988; 254: H102-H114

24. SHEEHAN FH, DOERR R, SCHMIDT WG, BOLSON EL, UEBIS R, VON ESSEN R, EFFERT S, DODGE HT: Early recovery of left ventricular function after thrombolytic therapy for acute myocardial infarction: An important determinant of survival. J Am Coll Cardiol 1988; 12: $289-300$

25. TOUCHSTONE DA, BELLER GA, NYGAARD TW, TEDESCO C, KAUL S: Effect of successful intravenous reperfusion therapy on regional myocardial function and geometry in humans: A tomographic assessment using two-dimensional echocardiography. J Am Coll Cardiol 1989; 13: $1506-1513$

26. PATEL B, KLONER RA, PRZYKLENK K, BRAUNWALD E: Postischemic myocardial "stunning": A clinically relevant phenomenon. Ann Intern Med 1988; 108: 626-628 